\title{
Low-cost deformable mirrors: technologies and goals
}

\author{
G. Vdovin ${ }^{a, b}$, M. Loktev ${ }^{b}$, A. Simonov ${ }^{a}$ \\ ${ }^{a}$ TU Delft, Mekelweg 4, 2628 CD, Delft, The Netherlands \\ ${ }^{b}$ OKO Technologies, PO Box 581, 2600 AN Delft, The Netherlands
}

\begin{abstract}
New applications of adaptive optics, especially in the potentially mass markets such as laser optics, imaging and medicine, require development of new components with high quality and low price. These requirements are equally applicable to wavefront sensors, wavefront reconstructors and wavefront correctors. The whole concept of adaptive optics as a science-intensive technology needs to be altered, to facilitate low-cost and service-free deployment and user-unaware exploitation.
\end{abstract}

As an example of a technology, that has a good low-cost potential, we describe the technology of piezoelectric deformable mirrors with actuators based on the transversal piezoelectric effect, as an inexpensive alternative to the deformable mirrors with stacked actuators.

Keywords: Adaptive optics, deformable mirror, wavefront sensing

\section{INTRODUCTION}

Deformable mirrors are complex opto-electro-mechanical devices. Expensive materials, strict tolerances and tough requirements to the parameters such as speed, linearity, thermal stability, temporal stability, etc., make them expensive. Traditionally, to satisfy these requirements, deformable mirrors were designed and fabricated with a great degree of technical reliability and resource overkill, using the best and the most expensive and precise technologies. These mirrors are similar to hand-assembled race cars, with price tags matching.

We believe that a great deal of design and technological optimization is applicable to the technology of deformable mirrors. Such an optimization will result in a robust, stable and low-cost adaptive optics with a wide range of application. These mirrors would be rather associated with inexpensive reliable mass-produced multi-functional family cars.

\section{OVERVIEW OF THE DM TECHNOLOGIES}

There is no such thing as a "generic" deformable mirror. ${ }^{1}$ Every technology limits the achievable corrector parameters with respect to the number of actuators, the correction stroke, speed of response, linearity etc. ${ }^{2}$ On the other hand, the arsenal of technologies applicable to building the wavefront correctors is rather limited. The most important of them are depicted in the Table 1.

Although, the available technologies are limited, it is possible to formulate some general requirements to an ideal wavefront corrector. Technically such a corrector should have potentially a very large number of degrees of freedom. The amplitude and the geometry of its influence functions should be matched to the Karhunen-Loeve functions, statistically calculated for the wavefronts to be corrected. ${ }^{2}$ Finally, the speed of response should be sufficient to cover the whole temporal spectrum of the corrected aberration.

Both bimorph ${ }^{3}$ and membrane ${ }^{4}$ (including micromachined membrane ${ }^{5}$ ) mirrors are suitable for correction of low-order aberrations in a wide frequency spectrum. Unfortunately, limited curvature of the mirror surface limits the number of control channels. It is possible to make mirrors with a very large numbers of

Further author information: Send correspondence to Gleb Vdovin, E-mail: gleb@okotech.com, http://www.okotech.com

Fax: +31-15-2574233

Advanced Wavefront Control: Methods, Devices, and Applications III, edited by Mark T. Gruneisen, John D. Gonglewski, Michael K. Giles, Proceedings of SPIE Vol. 5894 (SPIE, Bellingham, WA, 2005) 0277-786X/05/\$15 - doi: 10.1117/12.621042 
Table 1. Existing technologies for deformable mirrors.

\begin{tabular}{|c|c|c|}
\hline Mirror & Construction and actuators & Characteristics \\
\hline $\begin{array}{l}\text { 1. Deformable } \\
\text { substrate or } \\
\text { "continuous } \\
\text { phase-sheet" }\end{array}$ & $\begin{array}{l}\text { Discrete axial piezoelectric (PZT) or electrostrictive (PMN) actuators produce } \\
\text { local displacement of the substrate: } \\
\text { - Thing glass sheet with bonded actuators } \\
\text { - Modal response with very large number of actuators possible } \\
\text { Shape of the mirror: } \\
r(x, y)=\sum_{i} V_{i} r_{i}(x, y) \text {, } \\
\text { where } V_{i} \text { is the voltage applied to the } i \text {-th actuator; } r_{i}(x, y) \text { is the i-th } \\
\text { influence function. Can be designed with a wide variety of actuators }\end{array}$ & $\begin{array}{l}\text { 1. Number of actuators: } \\
\text { up to } 1000 \text {, potentially up } \\
\text { to } \sim 100000 \\
2 \text {. Inter-actuator spacing: } \\
\text { 2-10mm; } \\
\text { 3. Electrode geometry: } \\
\text { rectangular or hexagonal; } \\
\text { 4. Voltage: few hundred } \\
\text { V; } \\
\text { 5. Stroke (depends on } \\
\text { actuators): up to } \sim 10 \\
\text { microns; } \\
\text { 6. Resonant frequency: } \\
\text { few kHz; } \\
\text { 7. Cost: high. }\end{array}$ \\
\hline 2. Bimorph & $\begin{array}{l}\text { Shape of the mirror is determined by: } \\
\nabla^{4} r(x, y)=-A \nabla^{2} V(x, y) \\
\text { where } A \text { is the constant related with the properties of piezoelectric material } \\
\left(A \propto d_{13}\right), V \text { is the voltage distribution in the plane of the PZT. In terms of } \\
\text { spatial spectrum: } \widetilde{r}(\vec{k}) \propto|\vec{k}|^{-2} V(\vec{k}) \text {. So, bimorph DMs are well matched to } \\
\text { compensate atmospheric turbulence }\left(S(\vec{k}) \sim|\vec{k}|^{-11 / 6}\right) \text {. }\end{array}$ & $\begin{array}{l}\text { 1. Number of actuators: } \\
13-85 \text {; } \\
\text { 2. DM size: } \sim 30-200 \mathrm{~mm} \text {; } \\
\text { 3. Electrode geometry: } \\
\text { radial; } \\
\text { 4. Voltage: few hundred } \\
\text { V; } \\
\text { 5. Stroke: few microns: } \\
\text { 6. Resonant frequency: } \\
\text { more than } 500 \mathrm{~Hz} \text {; } \\
\text { 7. Cost: moderate. }\end{array}$ \\
\hline 3. Membrane & $\begin{array}{l}\text { Membrane (including micromachined membrane) with global curvature } \\
\text { controlled by an array of electrostatic actuators: } \\
\text { Membrane } \\
\text { Shape of the mirror is determined by: } \\
\nabla^{2} r(x, y)=\frac{q\left(V_{i}\right)}{D}, q\left(V_{i}\right) \sim\left\{V_{i}^{2}\right\}, \quad \text { where } q \text { is the voltage-dependent } \\
\text { distributed loading and } D \text { is the coefficient describing the flexural rigidity of the } \\
\text { membrane. }\end{array}$ & $\begin{array}{l}\text { 1. Number of actuators: } \\
\text { up to } 10^{3} \text {, increasing the } \\
\text { number of actuators } \\
\text { reduces the stroke per } \\
\text { actuator; } \\
\text { 2. Electrode geometry: } \\
\text { rectangular or hexagonal; } \\
\text { 4. Voltage: few hundred } \\
\mathrm{V} \text {; } \\
\text { 5. Stroke: few microns, } \\
\text { limited by the mirror } \\
\text { curvature and the actuator } \\
\text { size. } \\
6 . \text { Resonant frequency: } \\
\text { several kHz; } \\
\text { 7. Cost: moderate. }\end{array}$ \\
\hline
\end{tabular}




\begin{tabular}{|c|c|c|}
\hline 4. Segmented & $\begin{array}{l}\text { A technique in which many smaller elements (tip, tilt and piston actuators) are } \\
\text { built and then actively controlled to conform to the shape of the required large } \\
\text { mirror: } \\
\text { - Made of separate segments with small gaps; } \\
\text { - Each segment has } 1-3 \text { actuators. } \\
\text { The construction is usually used for large telescopes. Actuators directly control } \\
\text { the shape of the mirror. }\end{array}$ & $\begin{array}{l}\text { 1. Number of actuators: } \\
\text { up to } \sim 10^{2} \text {; } \\
\text { 2. Elements geometry: } \\
\text { sector-shaped segments } \\
\text { or hexagonal segments; } \\
\text { 4. Control signals: } \\
\text { depend on the actuator } \\
\text { type: typically, precision } \\
\text { electromechanical drivers } \\
\text { are employed; } \\
\text { 5. Stroke: may be as large } \\
\text { as needed; } \\
\text { 6. Response time: } \\
\sim \text { several hindered ms; } \\
\text { 7. Cost: very high. }\end{array}$ \\
\hline 5. Liquid crystal & $\begin{array}{l}\text { Liquid crystal (LC) based spatial light modulators. } \\
\text { Phase modulation stems from the orientation of LC molecules in the presence of } \\
\text { applied electric field. } \\
\text { Phase profile is determined by the orientation of LC molecules that is controlled } \\
\text { by applied electric field. Typically, the amplitude of phase shift does not exceed } \\
2 \pi \text { and the mirror operates in a "wrapped" mode. A number of approaches } \\
\text { developed for a modal operation of the LC correctors. }\end{array}$ & $\begin{array}{l}\text { 1. Number of actuators: up } 1 \\
\text { 2. Electrode geometry: } \\
\text { rectangular or hexagonal; } \\
\text { 3. Voltage: } \sim 10 \mathrm{~V} \text { at a } \\
\text { frequency of several } \mathrm{kHz} \text {; } \\
\text { 4. Effective stroke: one } \lambda \\
\text { to several } \lambda \\
\text { 5. Response time: } \\
\text { typically }>10 \mathrm{~ms} \text {; } \\
6 . \text { Cost: moderate as the } \\
\text { technology is similar to } \\
\text { LCDs, potential to be } \\
\text { inexpensive; } \\
\text { 7. Polarization sensitive, } \\
\text { strong dispersion, slow } \\
\text { response, temperature } \\
\text { sensitive. }\end{array}$ \\
\hline $\begin{array}{l}\text { 6. MEMS } \\
\text { (micro-electro- } \\
\text { mechanical } \\
\text { systems) }\end{array}$ & $\begin{array}{l}\text { MEMS-based DMs are made by micro-lithography and semiconductor batch } \\
\text { processing, in a way similar to electronic chips. } \\
\text { Small mirror elements are deflected by electrostatic forces. } \\
\text { Overall profile of the DM is directly controlled by array of micro mirrors. } \\
\text { the remaining problems of deformable mirrors utilizing current MEMS } \\
\text { difficulties with multilayer coating, difficulties with integration of large arrays } \\
\text { of high voltage drivers. }\end{array}$ & $\begin{array}{l}\text { 1. Number of actuators: } \\
\text { up to } 10^{5} \div 10^{6} \text {; } \\
2 \text {. Electrode geometry: } \\
\text { rectangular, hexagonal } \\
\text { etc. ; } \\
\text { 3. Voltage: several } \mathrm{V} \text {; } \\
4 \text {. Stroke: up to several } \\
\mu \mathrm{m} \text {; } \\
5 . \text { Response time: } \\
\text { expected to be less than } \\
10 \mu \mathrm{s} \text {; } \\
6 . \text { Cost: potential to be } \\
\text { very inexpensive - } \\
\text { estimation } \$ 10 \text { per } \\
\text { actuator. }\end{array}$ \\
\hline
\end{tabular}


channels, but the maximum response of each channel will be just a fraction of a wavelength, insufficient for the majority of applications.

Liquid crystal correctors ${ }^{6,7}$ have demonstrated their low-cost potential and can be used in transmission mode. Their serious drawbacks such as polarization sensitivity, slow response, strong dispersion and limited temperature range, limit the areas of possible applications.

MEMS mirrors ${ }^{8,9}$ have a huge future potential in scientific instrumentation, military and consumer markets. They are small, inexpensive, have very fast response and can be mass produced. The technology has a very good compatibility with electronics, allowing for higher degrees of integration. The drawbacks include small size (many applications require large apertures), limited range of available coatings, inferior surface quality and difficulties of high-voltage control on a micro-scale. Diffraction naturally limits the size of free-space optical elements, imposing certain limits on the miniaturization of the adaptive optics.

The emerging generations of astronomical AO systems will call for the development of deformable mirrors with clear apertures in the range of 5 to $100 \mathrm{~cm}$ with thousands to millions of control channels. Clearly, to make these technologies affordable, the existing approaches should be modified.

Currently available technologies do not allow the manufacturing of relatively low-cost high-order DMs with high dynamic range and operating frequencies of several $\mathrm{kHz}$. The main impediment for the realization of a high-order low-cost DM and difficulty of implementation of a massive inexpensive actuator array. Optimized driving electronics and effective control algorithms should be also elaborated for them to allow real-time operation as much as 1000 to 1000000 degrees of freedom.

Deformable mirrors with stacked ceramic push-pull actuators ${ }^{10,11}$ are free from the stroke limitations, as each actuator is able to produce a considerable stroke, limited only by the mechanics of the actuator and the flexible reflective face sheet. These mirrors can be fabricated with actuator pitch of several millimeters providing a good scale match for modern astronomical and imaging optics and can be fabricated with thousands of actuators, on a very high price, exceeding $\$ 1000$ per actuator.

Stacked actuators are characterized by very high stiffness, good reliability, relatively low driver voltages (up to 100V) and high capacitance. Since a very thin layers of material are used, these actuators are sensitive to humidity. Minimum price of $\$ 100$ per actuator makes deformable mirrors rather expensive.

Driver electronics for these actuators is rather expensive, as both high voltages and large currents should be commutated with high speed to secure quick re-charging of high capacitance actuators.

As a prompt conclusion, we can state that deformable mirrors with push-pull actuators and continuous faceplate provide a good solution to modern $\mathrm{AO}$ problems, have very good potential for scalability and suffer from very high costs, caused by expensive actuators and complex and bulky drivers. In the next sections we will describe an approach, that allows fabrication of inexpensive deformable mirrors with push-pull lower-power actuators based on the transversal piezoelectric effect.

\section{COMPARISON OF THE DIRECT AND THE TRANSVERSAL PIEZOELECTRIC EFFECTS}

The piezoelectric effect, as used in actuators, is the generation of a mechanical strain in response to a voltage. The piezoelectric effect is a linear effect (unlike the electrostrictive effect, which is quadratic) is present in non-centrosymmetric crystalline materials and tertain types of ceramics. To produce a piezoelectric actuator, the ceramics is poled in the external electric field. Significant polarization $P_{0}$ remains in the material after the external field is removed.

To produce a direct mechanical actuation, the external field $E$ applied to the piezo actuator should be parallel to the vector of internal polarization $P_{0}$. The actuator size changes under the action of the external field.

The actuator dimensions change not only along the field, but also in the orthogonal to the field direction - the so called transversal piezoeffect. 
The relative deformation $\epsilon=\Delta l / l$ in any direction is given by a relation:

$$
\epsilon=d E+e \sigma
$$

where $d$ is the modulus of the piezoelectric coefficient, $E$ is the field, $e=1 / Y$ where $\mathrm{Y}$ is the young's modulus and $\sigma$ is the stress.

For the deformation along the field we have to take $d=d_{33}$, for the transveral piezoeffect we should take $d=d_{31}$. The field $E$ should not exceed some maximum value $E_{c}$ to avoid the de-polarization of the actuator followed by loss of piezoelectric properties.

Let us derive the main parameters of a piezo actuator. Multiplying both parts of (1) by $l$, we obtain:

$$
\Delta l=d_{33} V-f / k
$$

where $V$ is the applied voltage, $k=S Y / l$ is the actuator stiffness ( $S$ is the cross section area) and $f$ is the force applied to the actuator, $f=-\sigma S$. If no control voltage is applied, the actuator is equivalent to a elastic rod with stiffness $k$. The elongation of unloaded actuator is equal to:

$$
\Delta l=d_{33} V
$$

If the actuator is loaded, the elongation is always smaller than defined by (3).

The sensitivity of the actuator to the external control voltage $\Delta l / V$ does not depend on the actuator length and depends only on its piezo modulus $d_{33}$ and the applied voltage. High control voltage is a serious drawback of piezoelectric actuators. To reduce the control voltage, a piezoelectric stacks are used - see Fig. 1. In this case $\Delta l=n d_{33} V$ where $n$ is the number of layers. This type of piezoceramic actuator can have a very high stiffness, as the layers can have large area.

In case when a sensitive and inexpensive actuator is needed, the transversal piezoelectric effect can be used. In this case, instead of (2) we have:

$$
\Delta l=V d_{31} l / h-f / k
$$

where $V=E h, h$ is the thickness of the element (for instance the thickness of the tube wall in the case of tubular actuator) and $l$ is the length of the actuator. Although $d_{31}$ is usually only a half of $d_{33}$, much larger elongations can be achieved because of large ratio $l / h$ which can easily reach $30 \ldots 50$. Maximum displacement is given by $\Delta l_{\max }=d_{31} l E_{c}$. These actuators demonstrate relatively low stiffness, compared to the stacked actuators. Actuators based on the transversal effect are usually made in the form of tubes, with the control voltage applied between the internal and external walls.

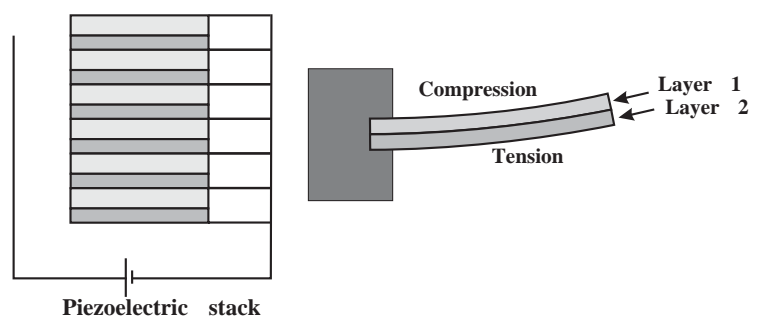

Figure 1. Two multi-layer piezoelectric configurations.

Transversal piezoelectric actuators do not require complex co-firing procedures as they do not have any layered structure and can be fabricated from the bulk of a ceramic material. Moreover, the industry offers low-cost ceramic actuators - see Fig. 4 - that can be directly used for fabrication of piezoelectric deformable mirrors. 

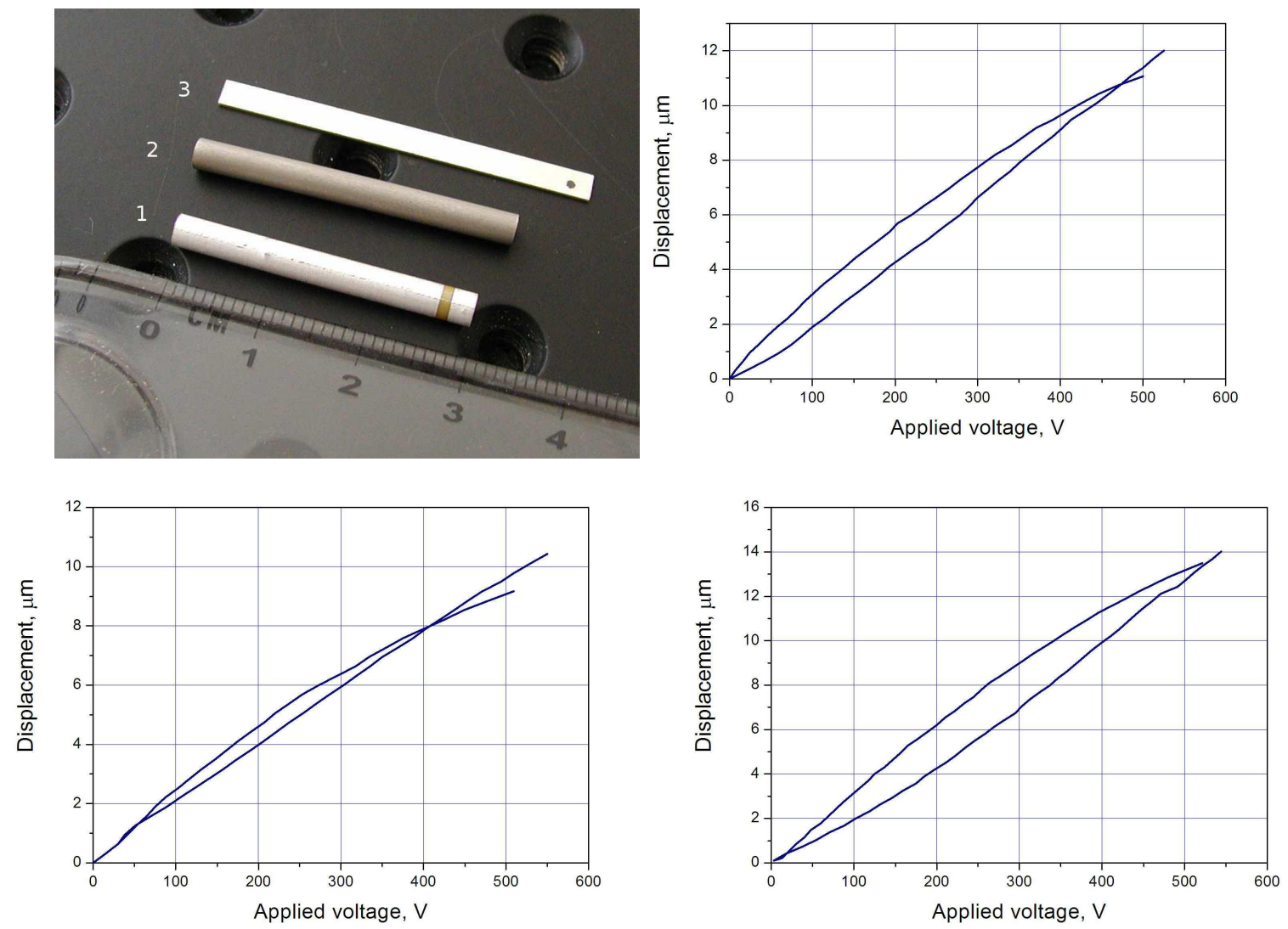

Figure 2. Transversal piezoelectric actuators from three different manufacturers (top left 1: PI; 2: Morgan Electroceramic; 3: Elma) and correspondent measured hysteresis curves. Top right corresponds to manufacturer 1, bottom left - manufacturer 2 .

The energy dissipation $P$ per actuator with capacitance $C$ and control voltage $U$ can be estimated as $P=f C U^{2} / 2$ where $f$ is the driving frequency. For an actuator based on the transversal piezoeffect with parameters $C=5 \cdot 10^{-9} \mathrm{~F}, U=400 \mathrm{~V}$ and $f=1000 \mathrm{~Hz}$, we obtain $P=400 \mathrm{~mW}$, while for a stacked actuator with $C=1.5 \cdot 10^{-6} \mathrm{~F}, U=100 \mathrm{~V}$ and $f=1000 \mathrm{~Hz}$, we obtain $P=7.5 \mathrm{~W}$. The power dissipation for a stacked piezoactuator is $\sim 19$ times higher than for a transversal piezo actuator. While making this comparison, we need to take into account that the stiffness of the stacked actuator is also much higher.

\section{DEFORMABLE MIRRORS WITH ACTUATORS BASED ON THE TRANSVERSAL PIEZOEFFECT}

To demonstrate the applicability of piezoactuators based on the transversal piezoeffect, we developed and assembled two low-cost deformable mirrors, with 19 and 37 actuators. Both mirrors have actuators placed in a hexagonal grid. The pitch of the grid is $6 \mathrm{~mm}$ for the 19-ch mirror and $4.3 \mathrm{~mm}$ for the 37-ch mirror. Both mirrors have aperture of $30 \mathrm{~mm}$. The technical specifications a 37-ch mirror are shown in the table 2 .

Actuators based on the transversal piezo effect have relatively low stiffness, therefore the stiffness of the reflective faceplate should be matched to the stiffness of the actuator. Using matched glass and quartz plates, we obtained up to $+/-1.5 \mu \mathrm{m}$ profile difference between adjacent actuators with a response time of better than $250 \mu \mathrm{s}$.

Fabrication of thin reflective plates with good planarity represents a rather unpleasant technological problem. The situation is complicated even more by the curvature, induced by the stress in the optical 


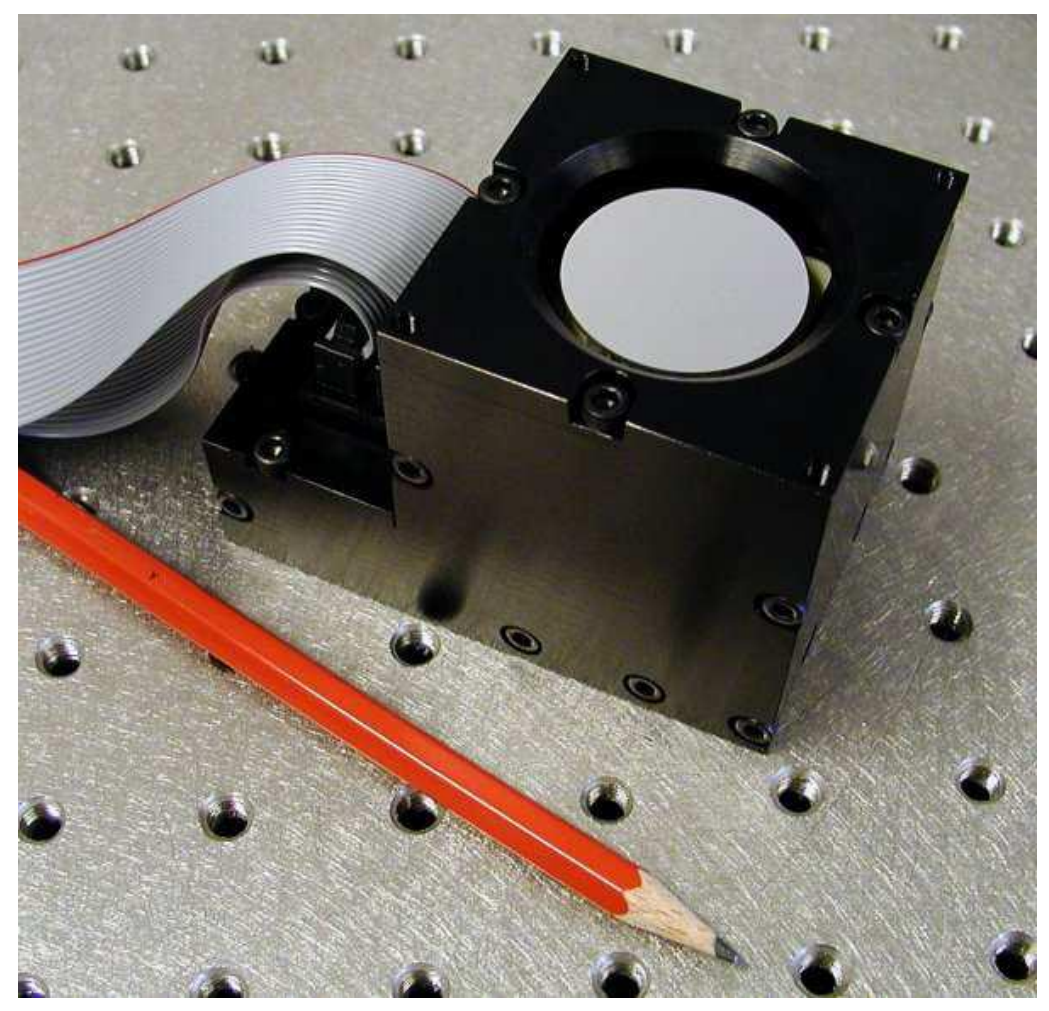

Figure 3. Deformable mirror with 37 piezoelectric actuators based on transversal piezo effect.

Table 2. Technical data of the $37 \mathrm{ch} 30 \mathrm{~mm}$ piezoelectric deformable mirror

\begin{tabular}{|l|l|}
\hline \hline Parameter & Value \\
\hline \hline Aperture shape & circular $30 \mathrm{~mm}$ in diameter \\
\hline Mirror coating & $\mathrm{Al}+\mathrm{MgF}_{2}$ (can be coated with HR multi-layer stack) \\
\hline Actuator voltages & $-0+400 \mathrm{~V}$ (with respect to the ground electrode) \\
\hline Number of actuators & 37, with $4.3 \mathrm{~mm}$ pitch \\
\hline Actuator capacitance $C_{a}$ & $5 \mathrm{nF}$ \\
\hline Initial RMS deviation from reference sphere & less than $1.3 \mu \mathrm{m}$ \\
\hline Main initial aberration & concave sphere with $\mathrm{R} \sim 30 \mathrm{~m}$ \\
\hline Frequency range & $0 \ldots .2000 \mathrm{~Hz}$ with full amplitude, \\
\hline Maximum stroke & $-8 \mu \mathrm{m}$ at $+400 \mathrm{~V}$ \\
\hline Inter-actuator stroke & $+/-1.5 \mu \mathrm{m}$ \\
\hline
\end{tabular}

coating. We decided to allow the mirror surface to have a slight curvature, taken that the aberration with respect to the nearest spherical reference is small.

Typical interferometric patterns, obtained for the 37ch deformable mirror are shown in Fig. 4

The typical power consumption with steady voltages on all 37 actuators does not exceed $8 \mathrm{~W}$, dissipated in the control unit, consisting of two 20-ch high-voltage amplifier boards of a eurocard $(10 \mathrm{x} 15 \mathrm{~cm})$ size. The power dissipation reaches $40 \mathrm{~W}$, when all actuators of the mirror are driven with maximum amplitude with a frequency of $1 \mathrm{kHz}$.

Fabrication technology used to assemble these mirrors, allows significant increase of the number of actuators, preserving the pitch in the range of 4.3 to $5 \mathrm{~mm}$. Since a very large number of external amplifiers and corresponding electrical connections reduces the reliability of the system, we decided to investigate the 

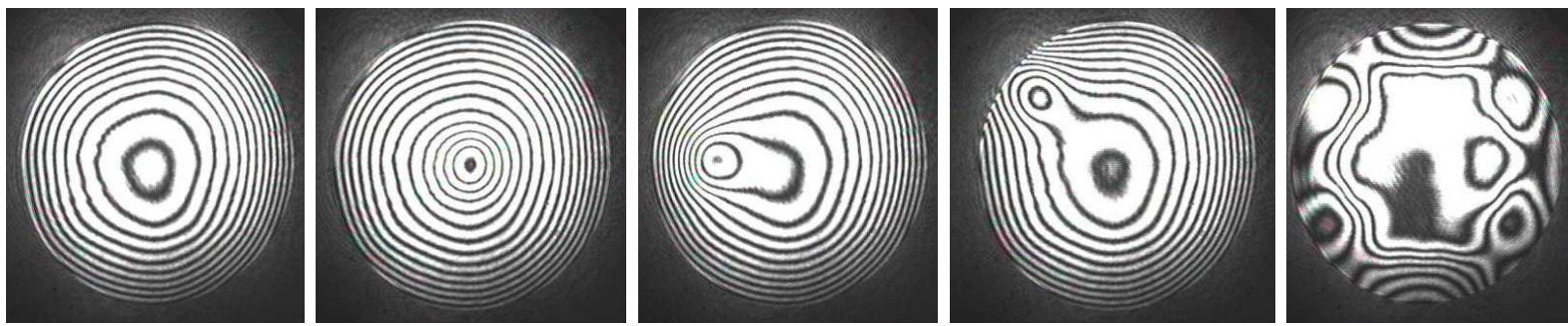

Figure 4. Interferometric patterns corresponding to the responses of the $37 \mathrm{ch}$ piezoelectric deformable mirror.

possibility of multiplexed control, with further steps directed to integration of the multiplexer electronics within the deformable mirror.

\section{MULTIPLEXING CONTROL}

As a demonstrator, a 12-channel piezoelectric DM with a clear aperture of $25.4 \mathrm{~mm}$ was fabricated on the basis of technology described above. The mirror used $3.2 \times 30 \mathrm{~mm}$ tubular PZT actuators positioned in a rectangular grid with a $7 \mathrm{~mm}$ pitch. The measured capacitance was $12 \mathrm{nF}$ and the discharge time (due to the leakage) exceeds $30 \mathrm{~s}$. The DM has a full stroke of $\sim 7 \mu \mathrm{m}$ with a hysteresis not exceeding $9 \%$ and an inter-actuator stroke of $\sim 2.5 \mu \mathrm{m}$, all measured in the 0 to $300 \mathrm{~V}$ range of control voltages.

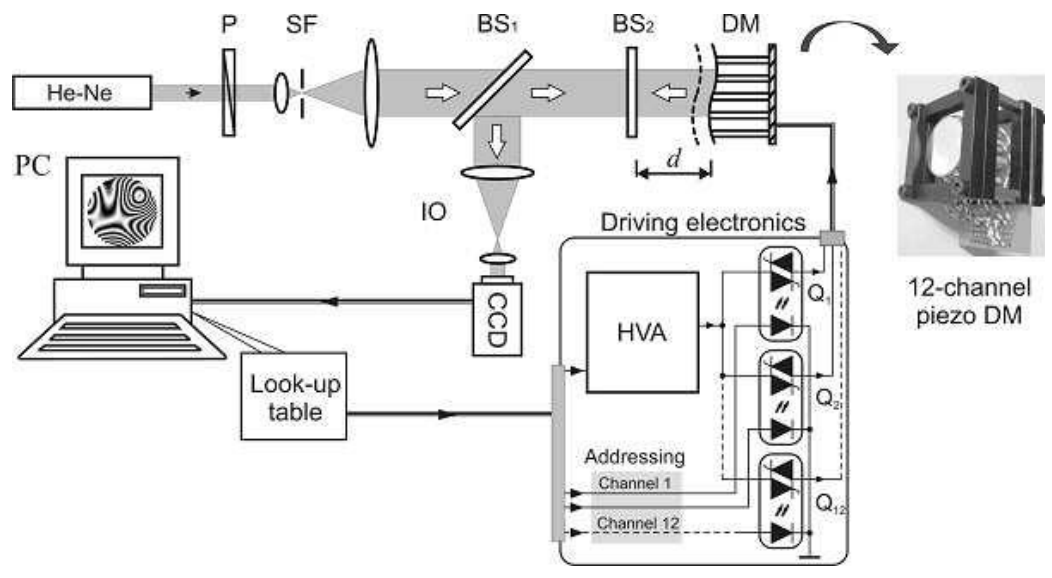

Figure 5. Experimental setup for characterization of the multiplexed control. P, polarizer; SF, spatial filter; BS1, BS2, beam splitters; IO, imaging optics; DM, deformable mirror; HVA, high-voltage amplifier; $\mathrm{Q}_{1} \ldots \mathrm{Q}_{12}$, optically controlled triacs.

Fig. 5 shows the experimental setup used to characterize the multiplexed control of the mirror. In the experiment, we used a single high voltage amplifier to drive all 12 actuators of the mirror through a network of high-voltage switches.

The experiment consisted in setting the mirror actuators to a random static set of control voltages and then in increasing the multiplexing frequency to a limit at which the mirror shape becomes unstable. The surface stability is measured by observing a single interferometric fringe in a setup shown in Fig. 5 . The results of the experiment are shown in Fig. 6.

The performance of the mirror has been examined in the most severe conditions of multiplexing with a large voltage difference between adjacent DM actuators. Fig. 6 b shows the threshold frequency of multiplexing as a function of the voltage difference. The maximum inter-actuator stroke between all adjacent actuators is achieved with the following set of voltages $\{\max , 0, \max , 0, \max , 0, \max , 0, \max , 0, \max , 0\}$, applied to the actuators. As seen, stable operation with the maximum mechanical inter-actuator stroke of 

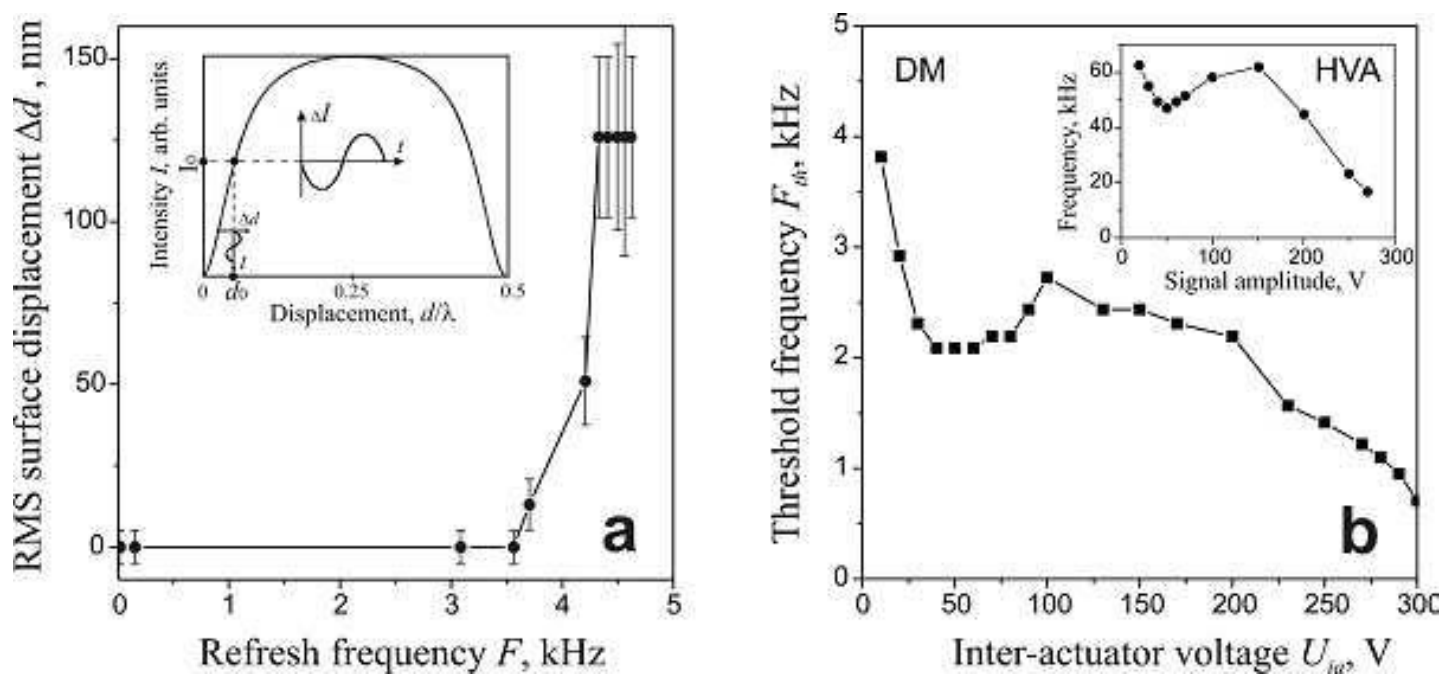

Figure 6. Multiplexing error (a): deviation of the DM figure from its static shape vs. multiplexing frame frequency. Inset shows the reflected intensity from the interferometer as a function of the surface displacement. (b) Threshold multiplexing frequency for the 12-channel DM vs inter-actuator voltage swing. Inset represents the output frequency-voltage response of the HVA loaded by a singe actuator.

$2.5 \mu \mathrm{m}$ (amplitude $300 \mathrm{~V}$ ) is possible with $F<700 \mathrm{~Hz}$, whereas the inter-actuator stroke of $0.1 \mu \mathrm{m}$ limits the frequency of stable multiplexing to $3.6 \mathrm{kHz}$.

The surface of the mirror is very stable at multiplexing frequencies that are lower than the limiting frequencies of the multiplexer.

The multiplexer includes a single optically controlled high-voltage triac switch per channel. Both the package size and the power dissipation were quite low, allowing for future integration of the multiplexing electronics into the package of the deformable mirror. Such a solution will strongly reduce the complexity of the controller and will be especially useful for deformable mirrors with a very large numbers of actuators.

\section{CONCLUSIONS}

We designed and implemented a low-cost continues faceplate deformable mirror with push-pull actuators based on transversal piezoelectric effect. The price of these actuators is at least one order of magnitude lower than the price of traditional stacked actuators. They dissipate at least 10 times less power in the controller. Low power dissipation allows for high speed efficient multiplexing of the mirror actuators: we have demonstrated stable control with multiplex coefficient of 12 at refresh frequency in the range of $700 \mathrm{~Hz}$ to $3.5 \mathrm{kHz}$, depending on the amplitudes of the control voltages. Simple switches used allow for future integration of the multiplexing circuitry within the deformable mirror.

\section{REFERENCES}

1. R. H. Freeman and J. E. Pearson, "Deformable mirrors for all seasons and reasons," Applied Optics 21, p. 580, 1982.

2. R. K. Tyson, Principles of adaptive optics, second edition, Academic Press, 1998.

3. Forbes, F. Roddier, G. Poczulp, C. Pinches, G. Sweeny, and R. Dueck, "Segmented bimorph deformable mirror," J. Phys. E: Sci. Instrum, pp. 402-405, 1989.

4. R. P. Grosso and M. Yellin, "Membrane mirror as an adaptive optical element," JOSA 67, p. 399, 1977.

5. G. Vdovin, P. Sarro, and S. Middelhoek, "Technology and applications of micromachined adaptive mirrors," Optical engineering 36, pp. 1382-1390, 1997. 
6. . G. D. Love, "Wave-front correction and production of zernike modes with a liquid rystal slm," Applied Optics 36, pp. 1517-1524, 1997.

7. A. F. Naumov and G. V. Vdovin, "Multichannel lc-based wavefront corrector with modal influence functions," Optics Letters 23(19), pp. 1550-1552, 1998.

8. T. Weyrauch, M. A. Vorontsov, T. G. Bifano, J. A. Hammer, M. Cohen, and G. Cauwenberghs, "Microscale adaptive optics: Wave-front control with a -mirror array and a vlsi stochastic gradient descent controller," Applied Optics 40, p. 4243, 2001.

9. M. K. Lee, W. D. Cowan, B. M. Welsh, V. M. Bright, and M. C. Roggemann, "Aberration correction results from a segmented microelectromechanical deformable mirror and a refractive lenslet array," Optics Letters 23, p. 645, 1998.

10. M. A. Ealey and J. Wellman, "Fundamentals of deformable mirror design and analysis," in Proc. SPIE, 1167, pp. 66-84, 1989.

11. M. A. Ealey and J. A. Wellman, "Xinetics low-cost deformable mirrors with actuator replacement cartridges," 2201, pp. 680-687, 1994. 